\title{
Evaluation of the Prognostic Role of Absolute Monocyte Count, Lymphocyte Monocyte Ratio and Neutrophil Lymphocyte Ratio in Hodgkin Lymphoma: Single Center Experience
}

\author{
Yasemin BOLUKBASI ${ }^{1,2}$, Duygu SEZEN ${ }^{1}$, Sukran SENYUREK ${ }^{1}$, Serdar ORNEK $^{3}$, \\ Ugur SELEK ${ }^{1,2}$, Burhan FERHANOGLU ${ }^{4}$ \\ ${ }^{1}$ Koc University, Faculty of Medicine, Department of Radiation Oncology, Istanbul, TURKEY \\ ${ }^{2}$ The University of Texas, MD Anderson Cancer Center, Department of Radiation Oncology, Houston, TX, USA \\ ${ }^{3}$ American Hospital, Department of Hematology, Istanbul, TURKEY \\ ${ }^{4}$ Koc University, Faculty of Medicine, Department of Hematology, Istanbul, TURKEY
}

\begin{abstract}
It is crucial to identify Hodgkin lymphoma $(\mathrm{HL})$ patients who may have resistance to treatment in order to choose the best risk adapted strategy at the time of diagnosis. We evaluated whether AMC (Absolute monocyte count), ALC (Absolute lymphocyte count), Lymphocyte/monocyte ratio (LMR), and Neutrophil Monocyte Ratio (NLR) were prognostic factors for HL. Sixty-five patients with classic Hodgkin Lymphoma were retrospectively reviewed. Initial peripheral blood tests were analyzed. Of the entire cohort, 96.9\% (63) had complete or partial remission. The median overall survival was 144 and median progression free survival was 130.7 months. The median AMC and LMR for all patients were $0.8 \times 10^{3} / \mathrm{UL}$ and 2.1. There were no statistically significant cut-off value for AMC, ALC and LMR. Median NLR was 3.9 and had a statistically significant cut-off value as $4.38(p=0.002)$. Mean overall survival time with $\mathrm{NLR}<4.38$ was 151.8 months versus 117.2 months for patients with $>4.38 \mathrm{NLR}(p=0.047)$. Mean progression free survival time was also significantly longer for patients with $N L R<4.38$ versus $>4.38$ ( 145.6 vs 102.8 months, $p=0.005$ ). Increased NLR sound to be a poor prognostic indicator in our cohort. NLR might be considered an additive parameter in management decision tree.
\end{abstract}

Keywords: Lymphoma, Monocyte, prognosis

\section{INTRODUCTION}

Hodgkin lymphoma has a relatively good prognosis compared to many other in lymphoma family; nevertheless, $10 \%$ to $30 \%$ of the patients was reported to be refractory to treatment or to relapse during the follow-up. ${ }^{1}$ Therefore, it is crucial to identify the Hodgkin lymphoma patients who might have resistance to treatment in order to choose the best risk adapted strategy at the time of diagnosis.

Various studies had been carried out in order to obtain easy-to-apply, affordable and reliable evaluation parameters about Hodgkin Lymphoma; Tumor associated macrophages (TAM), Absolute monocyte count (AMC), Lymphocyte/monocyte ratio (LMR) with different cut off values were assessed in retrospective studies as one of the easiest ways to provide an idea about systemic inflammation and tumor progression. ${ }^{2,3}$ Absolute monocyte count (AMC) has been also studied and found to have a significant relationship between increased AMC and unfavorable course of both HL and NHL in most studies. ${ }^{4-7}$ Similarly, the significant relationship between LMR value at the time of diagnosis and clinical outcome was also noted for both solid tumors and lymphomas. ${ }^{7-12}$ 
Increasing numbers of peripheral neutrophils, as the indicator of inflammatory response, have yet been reported as a sign of poor prognosis. ${ }^{13,14}$ All these analyses aimed to define a correlation between the patient's inflammatory response to cancer and immune response. ${ }^{5}$ The primary endpoint of our study was to evaluate whether AMC, ALC, LMR, and NLR were prognostic factors for HL in our cohort and to investigate whether there might be cut-off values to specify in our series.

\section{PATIENTS AND METHODS}

\section{Patients}

Between April 2008 and June 2017, a total of 65 patients treated with Hodgkin Lymphoma were retrospectively reviewed in this study. All patients met the following criteria: newly pathologically confirmed Hodgkin Lymphoma; age of 16 and older with available pretreatment hematologic parameters and detailed follow-up data, no previous history of malignancy and any oncological therapy; human immunodeficiency virus (HIV) negativity and also inclusion of other clinical studies. The patients were clinically staged according to the Ann Arbor Staging System. ${ }^{15}$ Patients with stage III - IV were stratified into the IPS-3 and IPS-7 scores. ${ }^{16,17}$ Although the IPS score is commonly used in advanced disease, the current classification was also performed for stage I-II in our study.

Baseline clinicopathological features like age, sex, histopathology, stage, presence of bulky disease, extra lymphatic lesions, bone marrow involvement or B symptoms were reviewed for all patients. $\mathrm{Pa}-$ tient and disease characteristics were listed in Table 1. Initial peripheral blood tests were provided and neutrophil, lymphocyte, monocyte, platelet, hemoglobin, C-reactive protein (CRP), sedimentation, albumin values were recorded for further consideration. All patients were stratified according to IPS-3 and IPS-7.

Treatment responses were evaluated with interim and post-treatment FDG-PET CT images. Routine follow-up was determined as every three months for the first 2 years, every six months between second and fifth years. Annually follow-up was undertaken since the fifth year.
The study was carried out with the written informed consent of patients in accordance with the ethical standards of the Declaration of Helsinki (Koc University Ethic Committee; 2021.195.IRB.068.)

\section{Statistical Analyses}

Statistical analysis was performed using the IBM Corp. Released 2019. IBM SPSS Statistics for Windows, Version 26.0. Armonk, NY: IBM Corp.). The normality of continuous variables were analyzed with Shapiro-Wilk test.

Overall survival was defined from the date of diagnosis to the last follow-up or death from any cause. Progression-free survival was calculated from the date of diagnosis to either the last follow-up or the occurrence of one of the following events: disease progression, relapse, or death from any cause. Mann-Whitney test and the KruskallWallis test were used for comparisons. Categorical variables were compared with the chi-square test and the exact Fisher test. Survival was evaluated by the Kaplan-Meier estimates and compared by using the log-rank test and the Cox proportional hazards model. We assessed the optimal cutoff for AMC, ALC, LMR, and NLR using the maximum $\log$-rank statistic and by means of receiver operating characteristic (ROC) curve analysis at 2-year follow-up.

Patients were divided according to IPS, 3 thereby 0 and 1-2-3 groups were created. Similarly IPS 7 categories were divided into 0-2 and 3-6 groups. Overall survival and progression free survival were compared between these groups separately for IPS 3 and IPS 7 scores with LogRank tests. Statistical significance was accepted when two-sided $\mathrm{p}$ value was lower than 0.05 .

\section{RESULTS}

The median age at diagnosis for the patients was 32 years (range, 16-84 years); $52.3 \%$ (34) were males, and $90.7 \%$ (59) was diagnosed with nodular sclerosis subtype. Other clinical characteristics are presented in Table 1.

Almost all of the patients (64 out of 65) were treated with standard 2-6 cycles of adriamycin, bleomycin, vinblastine and dacarbazine (ABVD) therapy regarding the initial stage. Only one pa- 


\begin{tabular}{|ll|}
\hline Table 1. Patient and disease characteristics & \\
\hline Characteristic & $\mathbf{n}(\%)$ \\
\hline Gender & \\
Male & $34(52.3)$ \\
Female & $31(47.7)$ \\
Age (range) & $16-84$ \\
Histologic type & \\
Nodular sclerosis & $59(90.7)$ \\
Mixed cellularity & $1(1.6)$ \\
Lymphocyte-rich & $4(6.1)$ \\
Lymphocyte-depleted & $1(1.6)$ \\
Ann-Arbor Stage & \\
I & $9(13.9)$ \\
II & $33(50.7)$ \\
III & $9(13.9)$ \\
IV & $14(21.5)$ \\
B symptoms present & $26(40.0)$ \\
Bulky disease present & $4(6.1)$ \\
IPS 3 & \\
0 & $45(69.2)$ \\
1-2-3 & $20(30.8)$ \\
IPS 7 & \\
O-2 & $54(83.1)$ \\
2-6 & $11(16.9)$ \\
ABVD & \\
CHOP with dose reduction & $62(95.5)$ \\
VEBEMP & $1(1.5)$ \\
BEACOPP & $1(1.5)$ \\
Radiotherapy & $33(50.7)$ \\
& \\
\hline
\end{tabular}

tient's treatment has been started with 2 cycles of bleomycin-etoposide-doxorubicin-cyclophosphamide-vincristine-procarbazine-prednisone (BEACOPP) and continued with 2 cycles of ABVD. Thirty-three patients (50.7\%) had radiation therapy as consolidation between the doses of $20 \mathrm{~Gy}$ and $30.6 \mathrm{~Gy}$.

Of the entire cohort, $84.6 \%$ of the patients had complete, only 8 patients has partial response at the interim PET-CT. Of those patients, only two did not have complete metabolic PET-CT response the end of the treatment. The median OS was 144 and median progression free survival was 130.7 months. A total of 6 deaths were recorded; of these, one of these deaths was from progressive disease and one was due to another primary cancer. The other deaths were not related to Hodgkin Lymphoma. The OS at 2 and 5 years was $96 \%$ and $91.9 \%$, respectively.

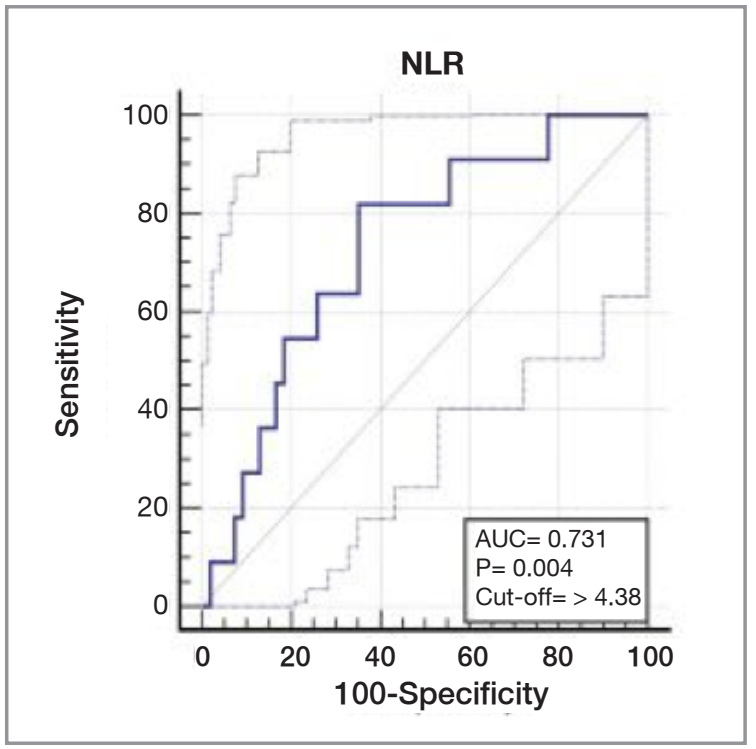

Figure 1. Discriminative cut-off value for the NLR was 4.38 (Sensitivity $81.8 \%$ and specificity 64.8 ; AUC values 0.731 , 95\% Cl: 0.580-0.882 $p=0.002) . A U C=$ area under the curve, $\mathrm{Cl}=$ confidence interval, NLR= neutrophil-to-lymphocyte ratio.

Median NLR was 3.9 (range: $0.05-46$ ) only NLR had a statistically significant cut-off value as 4.38 (95\% CI: $0.580-0.882, \mathrm{p}=0.002$ ). Mean overall survival time of the patients those NLR value was $<4.38151 .8$ months (95\% CI: 143.5-160), whereas patients with $>4.38 \mathrm{NLR}$ value have the mean overall survival time was 117.2 months $(95 \% \mathrm{CI}$ : 103.1-131.4) (Figure 1). They have statistical significant difference (Log Rank test $\mathrm{p}=0.047$ ).

Absolute monocyte count was taken from the pretreatment complete blood cell counts recorded at diagnosis of HL. The median AMC for all patients was $0.8 \times 10^{3} / \mathrm{UL}$ (range; 0.1-3.4 10 $10^{3} \mathrm{UL}$ ). A statistically significant cut-off value for AMC in the ROC curve analysis (95\% CI: 0.338-0.702 p= 0.82 ) could not be reached. Median ALC was derived from pretreatment complete blood cell count at diagnosis, and for all patients the median ALC was $1.6 \times 10^{3} / \mathrm{UL}$ (range $0.2-3.3 \times 10^{3} / \mathrm{UL}$ ). The LMR was obtained by dividing the ALC by the AMC taken from the complete peripheral blood count at diagnosis. The median LMR for all patients was 2.1 (range: $0.5-13$ ) according the analysis. Similarly there were no statistically significant cut-off value for ALC and LMR (95\% CI: 0,4580,$806 ; p=0.13$ and $95 \%$ CI: 0.484-0.807, $p=0.07$, respectively) (Figure 2). 

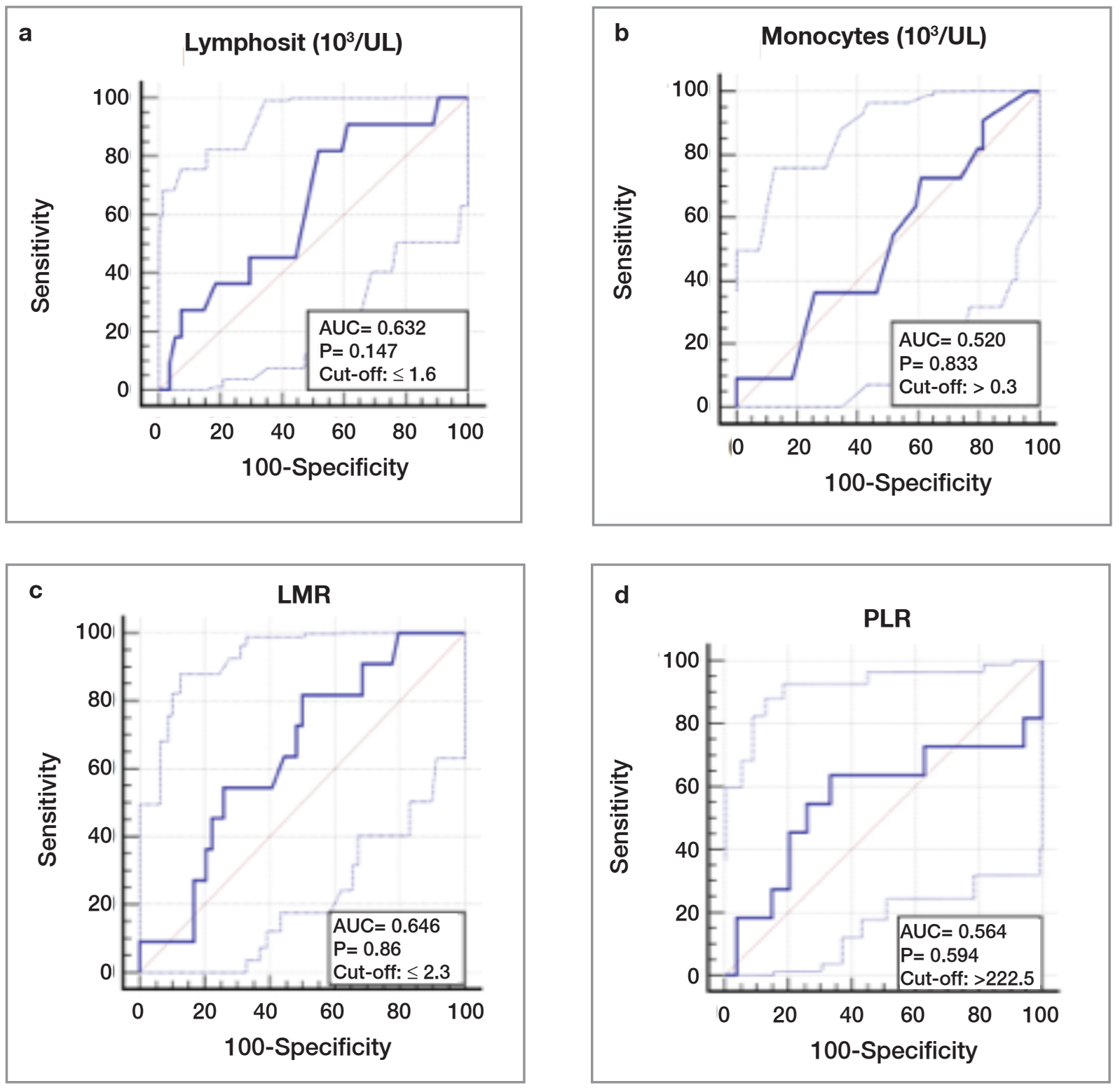

Figure 2. Significant discriminative cut-off value for (a) ALC and (b) AMC (c) LMR (d) PLR could not be demonstrated.

When evaluating by means of Progression Free Survival, patients also have statistically significant difference (Mean progression free survival time of patients those NLR value was < $4.38145 .6(95 \%$ CI: 135.6-155.6), whereas patients with $>4.38$ NLR value have the mean progression free survival time was 102.8 (95\% CI: 84.5-121.1). They have statistical significant difference (Log Rank test $\mathrm{p}=$ 0.005). Table 2)

Overall survival and progression free survival were compared between these groups separately for IPS
3 (IPS 30 vs 1-2-3) and IPS 7 (IPS 7 1-2 vs 3-6). Neither OS nor PFS difference were present for both IPS stratifications . (Table 3)

\section{DISCUSSION}

The relationship between hematological parameters and the prognosis of Hodgkin's lymphoma has been evaluated in several studies, due to the fact that being easy to be accessible as a part of the clinical routine; and especially AMC, LMR and NLR have been reported to be prognostic. ${ }^{13}$ It has 
International Journal of Hematology and Oncology

\begin{tabular}{|c|c|c|c|}
\hline os & Mean OS (m) & $95 \% \mathrm{Cl}$ & Log Rank test $p$ value \\
\hline$N L R<4.38$ & 151.8 & $143.5-160.0$ & 0.047 \\
\hline$N L R>4.38$ & 117.2 & $103.1-131.4$ & \\
\hline PFS & Mean PFS (m) & $95 \% \mathrm{Cl}$ & Log Rank test $p$ value \\
\hline$N L R<4.38$ & 145.6 & $135.6-155.6$ & 0.005 \\
\hline$N L R>4.38$ & 102.8 & $84.5-121.1$ & \\
\hline
\end{tabular}

been reported in several studies that monocytosis, lymphopenia or neutropenia are associated with poor prognosis and treatment resistance. ${ }^{6}$ Unfortunate is the lack of global consensus on cut-off values regarding these parameters. ${ }^{6,18,19}$ In our cohort, we have identified a cut off of NLR of 3.9 to be a significant predictor on both overall survival and progression free survival though IPS was not a player.

There is substantial data related to the monocytosis in Non-Hodgkin Lymphoma (NHL) and its effect on prognosis, while minimal data regarding Hodgkin lymphoma are available..$^{4,20,21}$ In a multicenter study that consists over one thousand diffuse large B cell lymphoma patients; the best AMC cut-off level was reported as $630 / \mathrm{mm}^{3}$. The 5-year overall survival for patients with counts below this cut-off was significantly longer than those with a count $>$ $630 \mathrm{~mm}^{3}$ (71\% vs 59\%, p=0.0002). ${ }^{22}$ In another study including $\mathrm{T}$ cell lymphoma, Bari et al retrospectively evaluated 94 cases and reported that the median OS for patients with monocyte value with $<0.8 \times 10^{9} / \mathrm{L}$ at diagnosis was longer than those with $>0.8 \times 10^{9} / \mathrm{L} 12$ (median OS was not reached after 9 years vs 12 months, respectively; $\mathrm{p}=0.003) .{ }^{23}$ In a large cohort study of Tadmor et al. with 1450 nodular sclerosis Hodgkin lymphoma patients; cutoff value of 750 cells $/ \mathrm{mm}^{3}$ for AMC was shown to have prognostic significance on PFS and OS while AMC more than 750 cells $/ \mathrm{mm}^{3}$ had an significantly adverse impact on outcome with both univariate $(\mathrm{p}<0.001)$ and multivariate analyses $(p=0.006)$. This study has importance due to high patient number as well as binationality and multicentricity of the treatment facilities. ${ }^{6}$ In our cohort, AMC was not statistically significant for any endpoints.

Lymphopenia is also an important indicator of host immunity and a well-defined parameter among IPS criteria in patients diagnosed with Hodgkin lymphoma. Lymphocyte count of less than 600 per cubic millimeter, a count that was less than 8 percent of the white-cell count, or both are known as adverse prognostic factors. ${ }^{16}$ In this context; LMR, the ratio of ALC to AMC, has been an attractive parameter for analysis. Tadmor et al reported that the best cutoff value for LMR was 2.1 in their 1450 patients diagnosed as nodular sclerosis HL. ${ }^{6}$ They also indicated that $\mathrm{LMR}<2.1$ was associated with the adverse prognostic factors in IPS and may be used to gain insight about tumor growth as well as host immunity and tumor microenvironment. We

\begin{tabular}{|c|c|c|c|c|c|}
\hline Variable & $\begin{array}{l}\text { Number of } \\
\text { patients }\end{array}$ & $\begin{array}{l}5 \text { years } \\
\text { Overall } \\
\text { Survival }\end{array}$ & $P$ value & $\begin{array}{l}5 \text { years } \\
\text { Disease free } \\
\text { survival }\end{array}$ & $P$ value \\
\hline IPS 3_0 & 45 & $95 \%$ & 0.18 & 88 & 0.93 \\
\hline IPS 3_1-3 & 20 & $85 \%$ & & 85 & \\
\hline IPS 7_1-2 & 54 & $92 \%$ & 0.07 & $84 \%$ & 0.19 \\
\hline IPS7_3-6 & 11 & $81 \%$ & & $72 \%$ & \\
\hline
\end{tabular}


could not reveal any significance related with LMR in our cohort.

Absolute neutrophil number serves as an indicator of the systemic inflammatory response to tumor. Inflammation had a significant role in tumor microenvironment. The NLR was defined as the division of the ANC by ALC at diagnosis. Marcheselli et al retrospectively analyzed 990 NS HL patients in terms of NLR at diagnosis and its effect on survival $^{13}$; and revealed a NLR cutoff of 6 with a statistical significant poor progression free $(84 \%$ vs $75 \%, \mathrm{p}<0.005)$ and overall survival ( $92 \%$ vs $88 \%$, $\mathrm{p}<0.005$ ) at five years in patients with NLR $>6$ in comparison to patients with NLR $<6$. This study was important for not only including a high number of homogenously staged pathologic subgroup of patients, but also delineating the difference in both univariate and multivariate analyses, and Marcheselli et al. clearly indicated NLR as an independent prognostic factor in NS HL in all stages of the disease. On the other hand, Keam et al reported their retrospective analysis of 447 DLBCL patients, and stated NLR $>3$ to be as an independent poor prognostic factor of PFS $(p<0.001)$ and OS ( $\mathrm{p}<0.001) .{ }^{24}$ NLR cutoff values as 3.0, 3,3 and 4.0 were reported in different solid tumor types. ${ }^{25-27}$ In our cohort, median NLR was 3.9 (range: 0.0546) and our statistically significant NLR cut off has been revealed to be 4.38 (95\% CI: $0.580-0.882$, $\mathrm{p}=$ 0.002); which was stratifying our patients with mean overall survival of 151.8 months $(95 \% \mathrm{CI}$ : 143.5-160) if < 4.38, and 117.2 months (95\% CI: 103.1-131.4) ( $\mathrm{p}=0.047)$ if NLR was $>4.38$. Progression Free Survival was also clearly statistically stratified with this cut off value; Mean progression free survival was 145.6 (95\% CI: 135.6-155.6) months if NLR < 4.38; and 102.8 (95\% CI: 84.5121.1) months if NLR $>4.38$, consistent with the related literature. Though IPS could not define a clear leveling in our cohort, NLR was able to declare the poor prognostic group of patients at diagnosis.

Our study has several important limitations, the most important being relatively small number of patients and being retrospective, as well as a low number of events in the follow up duration. Further analysis of pathological specimens of the patients with high NLR was also not possible in our cohort, where such analysis might probably provide more correlative information regarding the peripheral blood distribution and tumor microenvironment.

\section{Conclusion}

Our findings expressed that increased NLR is associated with poor prognosis in Hodgkin Lymphoma, which was interestingly a better predictor than IPS in this cohort, therefore we believe studies analyzing NLR need to be encouraged as an additive prognostic parameter in management decision tree to reveal the true potential. We await with much expectation for the future research including possible consensus cutoff values of AMC, LMR and, NLR in Hodgkin lymphoma.

\section{REFERENCES}

1. Ansell SM. Hodgkin lymphoma: 2018 update on diagnosis, risk-stratification, and management. Am J Hematol 93: 704$715,2018$.

2. Koh YW, Park CS, Yoon DH, et al. CD163 expression was associated with angiogenesis and shortened survival in patients with uniformly treated classical Hodgkin lymphoma. PLoS One 9: e87066, 2014.

3. Steidl C, Lee T, Shah SP, et al. Tumor-associated macrophages and survival in classic Hodgkin's lymphoma. N Engl J Med 362: 875-885, 2010.

4. Batty N, Ghonimi E, Feng L, et al: The absolute monocyte and lymphocyte prognostic index for patients with diffuse large B-cell lymphoma who receive R-CHOP. Clin Lymphoma Myeloma Leuk 13: 15-18, 2013.

5. Ho CL, Lu CS, Chen JH, et al. Neutrophil/lymphocyte ratio, Iymphocyte/monocyte ratio, and absolute lymphocyte count/ absolute monocyte count prognostic score in diffuse large B-cell lymphoma: Useful prognostic tools in the rituximab era. Medicine (Baltimore) 94: e993, 2015.

6. Tadmor T, Bari A, Marcheselli L, et al. Absolute monocyte count and lymphocyte-monocyte ratio predict outcome in nodular sclerosis Hodgkin lymphoma: Evaluation based on tata from 1450 patients. Mayo Clinic Proc 90: 756-764, 2015.

7. Porrata LF, Ristow K, Colgan JP, et al. Peripheral blood lymphocyte/monocyte ratio at diagnosis and survival in classical Hodgkin's lymphoma. Haematologica 97: 262-269, 2012.

8. Zhang S, Li M, Yuan F, et al. The predictive value of dynamic monitoring of peripheral blood lymphocyte to monocyte ratio in patients with extranodal NK/T cell lymphoma. Cancer Cell Int 19: 272, 2019. 
9. Belotti A, Doni E, Bolis S, et al. Peripheral blood lymphocyte/ monocyte ratio predicts outcome in follicular lymphoma and in diffuse large B-cell lymphoma patients in the rituximab era. Clin Lymphoma Myeloma Leuk 15: 208-213, 2015.

10. Lin ZX, Ruan DY, Li Y, et al. Lymphocyte-to-monocyte ratio predicts survival of patients with hepatocellular carcinoma after curative resection. World J Gastroenterol 21: 1089810906, 2015

11. Hutterer GC, Stoeckigt C, Stojakovic T, et al. Low preoperative lymphocyte-monocyte ratio (LMR) represents a potentially poor prognostic factor in nonmetastatic clear cell renal cell carcinoma. Urol Oncol 32: 1041-1048, 2014.

12. Nishijima TF, Muss HB, Shachar SS, et al. Prognostic value of lymphocyte-to-monocyte ratio in patients with solid tumors: A systematic review and meta-analysis. Cancer Treat Rev 41: 971-978, 2015.

13. Marcheselli R, Bari A, Tadmor T, et al. Neutrophil-lymphocyte ratio at diagnosis is an independent prognostic factor in patients with nodular sclerosis Hodgkin lymphoma: results of a large multicenter study involving 990 patients. Hematol Oncol 35: 561-566, 2017

14. Coffelt SB, Wellenstein MD, de Visser KE. Neutrophils in cancer: neutral no more. Nat Rev Cancer 16: 431-446, 2016.

15. Lister TA, Crowther D, Sutcliffe SB, et al. Report of a committee convened to discuss the evaluation and staging of patients with Hodgkin's disease: Cotswolds meeting. J Clin Oncol 7: 1630-1636, 1989

16. Hasenclever D, Diehl V. A prognostic score for advanced Hodgkin's disease. International Prognostic Factors Project on Advanced Hodgkin's Disease. N Engl J Med 339: $1506-$ 1514,1998

17. Moccia AA, Donaldson J, Chhanabhai M, et al. International Prognostic Score in advanced-stage Hodgkin's lymphoma: altered utility in the modern era. J Clin Oncol 30: 3383-3388, 2012.

18. Romano A, Parrinello NL, Vetro C, et al. Prognostic meaning of neutrophil to lymphocyte ratio (NLR) and lymphocyte to monocyte ration (LMR) in newly diagnosed Hodgkin lymphoma patients treated upfront with a PET-2 based strategy. Ann Hematol 97: 1009-1018, 2018.

19. Vassilakopoulos TP, Dimopoulou MN, Angelopoulou MK, et al. Prognostic implication of the absolute lymphocyte to $a b-$ solute monocyte count ratio in patients with classical Hodgkin lymphoma treated with doxorubicin, bleomycin, vinblastine, and dacarbazine or equivalent regimens. Oncologist 21: 343353, 2016.

20. Wilcox RA, Ristow K, Habermann TM, et al. The absolute monocyte count is associated with overall survival in patients newly diagnosed with follicular lymphoma. Leuk Lymphoma 53: 575-580, 2012
21. Wilcox RA, Ristow K, Habermann TM, et al. The absolute monocyte and lymphocyte prognostic score predicts survival and identifies high-risk patients in diffuse large-B-cell lymphoma. Leukemia 25: 1502-1509, 2011.

22. Tadmor T, Bari A, Sacchi S, et al. Monocyte count at diagnosis is a prognostic parameter in diffuse large B-cell lymphoma: results from a large multicenter study involving 1191 patients in the pre- and post-rituximab era. Haematologica 99: 125-130, 2014

23. Bari A, Tadmor T, Sacchi S, et al. Monocytosis has adverse prognostic significance and impacts survival in patients with T-cell lymphomas. Leuk Res 37: 619-623, 2013.

24. Keam B, Ha H, Kim TM, et al. Neutrophil to lymphocyte ratio improves prognostic prediction of International Prognostic Index for patients with diffuse large B-cell lymphoma treated with rituximab, cyclophosphamide, doxorubicin, vincristine and prednisone. Leuk Lymphoma 56: 2032-2038, 2015.

25. Motomura T, Shirabe K, Mano Y, et al: Neutrophil-lymphocyte ratio reflects hepatocellular carcinoma recurrence after liver transplantation via inflammatory microenvironment. J Hepatol 58: 58-64, 2013.

26. Ethier JL, Desautels D, Templeton A, et al. Prognostic role of neutrophil-to-lymphocyte ratio in breast cancer: a systematic review and meta-analysis. Breast Cancer Res 19: 2, 2017.

27. Faria SS, Fernandes PC, Jr., Silva MJ, et al. The neutrophilto-lymphocyte ratio: a narrative review. Ecancermedicalscience 10: 702, 2016.

\section{Correspondence:}

\section{Dr. Yasemin BOLUKBAS}

Koc Universitesi, Tip Fakultesi

Radyasyon Onkolojisi Anabilim Dali

Davutpasa Caddesi, No:4

34010, Topkapi, ISTANBUL / TURKEY

Tel: (+90-532) 6738757

e-mail: ybolukbasi@kuh.ku.edu.tr

\section{ORCIDs:}

Yasemin Bolukbasi 0000-0002-3170-5826

Duygu Sezen 0000-0002-4505-2280

Sukran Senyurek 0000-0003-3010-6132

Serdar Ornek 0000-0003-2602-1742

Ugur Selek 0000-0001-8087-3140

Burhan Ferhanoglu 0000-0002-4257-549X 